\title{
Origin of Anomalous Slip in Tungsten
}

\author{
C. Marichal, ${ }^{1,2}$ K. Srivastava, ${ }^{3}$ D. Weygand, ${ }^{3}$ S. Van Petegem, ${ }^{1}$ D. Grolimund,${ }^{4}$ P. Gumbsch,,${ }^{3,5}$ and H. Van Swygenhoven ${ }^{1,2}$ \\ ${ }^{1}$ Materials Science and Simulation, NUM/ASQ, Paul Scherrer Institut, CH-5232 Villigen PSI, Switzerland \\ ${ }^{2}$ Neutrons and X-rays for Mechanics of Materials, IMX, École Polytechnique Fédérale de Lausanne, CH-1012 Lausanne, Switzerland \\ ${ }^{3}$ Institute for Applied Materials IAM, Karlsruhe Institute of Technology, 76131 Karlsruhe, Germany \\ ${ }^{4}$ Beamline MicroXAS, Paul Scherrer Institut, CH-5232 Villigen PSI, Switzerland \\ ${ }^{5}$ Fraunhofer-Institut für Werkstoffmechanik IWM, Wöhlerstrasse 11, 79108 Freiburg, Germany
}

(Received 29 January 2014; published 7 July 2014)

\begin{abstract}
Low-temperature deformation of body-centered cubic metals shows a significant amount of plastic slip on planes with low shear stresses, a phenomenon called anomalous slip. Despite progress in atomistic modeling of the consequences of complex stress states on dislocation mobility, the phenomenon of anomalous slip remained elusive. Using in situ Laue microdiffraction and discrete dislocation dynamics in micrometer sized tungsten single crystals, we demonstrate the occurrence of significant anomalous slip. It occurs as a consequence of cross kinks, topological configurations generated by prior dislocation interactions. This clearly identifies anomalous slip as a multidislocation process and not a property of isolated dislocations. The cross-kink mechanism also explains the ambiguous reporting of anomalous slip traces in the past and directs us to ways of including anomalous slip in continuum crystal plasticity formulations.
\end{abstract}

DOI: 10.1103/PhysRevLett.113.025501

PACS numbers: 61.72.Bb, 61.72.Dd, 61.72.Lk, 62.20.F-

Plastic deformation of metals as the consequence of dislocation glide appears as a well-understood phenomenon which can be directly modeled by dislocation based simulations. Nevertheless, it is still not possible to systematically describe macroscopic plastic response as a consequence of dislocation motion. This is particularly so for bec metals where many of the relevant phenomena are not sufficiently understood. It is well established that the flow stress in bcc metals deformed at low temperature is governed by the mobility of $\langle 111\rangle$ screw dislocations. Usually dislocations glide on planes in which the shear stress resolved in the slip direction exceeds a critical value [1]. The stress state can be resolved onto each slip system by projecting it onto the slip direction and slip plane normal, commonly described by the Schmid factor. In bcc metals, screw dislocations have a nonplanar core structure $[2,3]$ which can make their glide susceptible to other stress components, usually called nonSchmid stresses [4]. While they can explain the tensioncompression asymmetry [5,6], for example, they cannot explain the experimental observation of a significant amount of plastic slip on slip systems with very low resolved shear stresses, a phenomenon called anomalous slip [7]. It was first reported in niobium [8] and later established to be a characteristic feature of low-temperature deformation of most bcc metals [9-12]. Past experiments indicate that anomalous slip occurs at small strains in molybdenum and tungsten $[9,11]$ and as a persistent mechanism in the absence of visible equal slip traces on the primary plane in niobium and tantalum $[10,12]$. To clarify the role of anomalous slip in tungsten, we performed in situ Laue diffraction experiments on single crystals and mimicked the experiment with discrete dislocation dynamics simulations.
Tungsten single crystals in the $\left[\begin{array}{lll}-1 & 5 & 10\end{array}\right]$ orientation were compressed during Laue diffraction at MicroXAS (Swiss Light Source). Details on the setup are given in [13] and [14]. The primary $(-101)[111]$ and the conjugate $(101)[-111]$ slip systems are expected to be the most active in this orientation. Figure 1(a) shows the orientation of the W crystal in stereographic projection as well as the relevant slip planes and directions. Table I in the Supplemental Material [13] details the pillar geometry and the Schmid factors together with the initial Laue peak profiles prior to deformation. The absence of any streaking indicates the absence of excess dislocations [14].

Figure 1(a) provides the stress-strain curves of two pillars, showing significant hardening. Pillar 1 is deformed until $16 \%$ strain reaching a flow stress of $2000 \mathrm{MPa}$, pillar 2 until 23\% strain reaching $1700 \mathrm{MPa}$. Such a difference in flow stress is not unusual for microcompression experiments [15]. Following the movement of diffraction spots during deformation provides information on the crystal rotation caused by dislocations slipping on particular planes [16-18]. The rotation directions expected for slip on the six $\{110\}$ planes are drawn on the Laue plots. Figure 1(b) shows the evolution of the $(-10-1)$ diffraction spot of pillar 2 . The corresponding stress levels are indicated with image numbers on the stress-strain curve: before loading (1), at the end of the elastic region (82), at 9\% total strain (123), just before unloading at $20 \%$ strain (145), and in the unloaded state. During elastic loading, the compression axis rotates slightly. This is ascribed to elastic distortion of the crystal and to initial unavoidable small misalignments between pillar and indenter [19]. At pattern 82 (900 MPa), a significant 

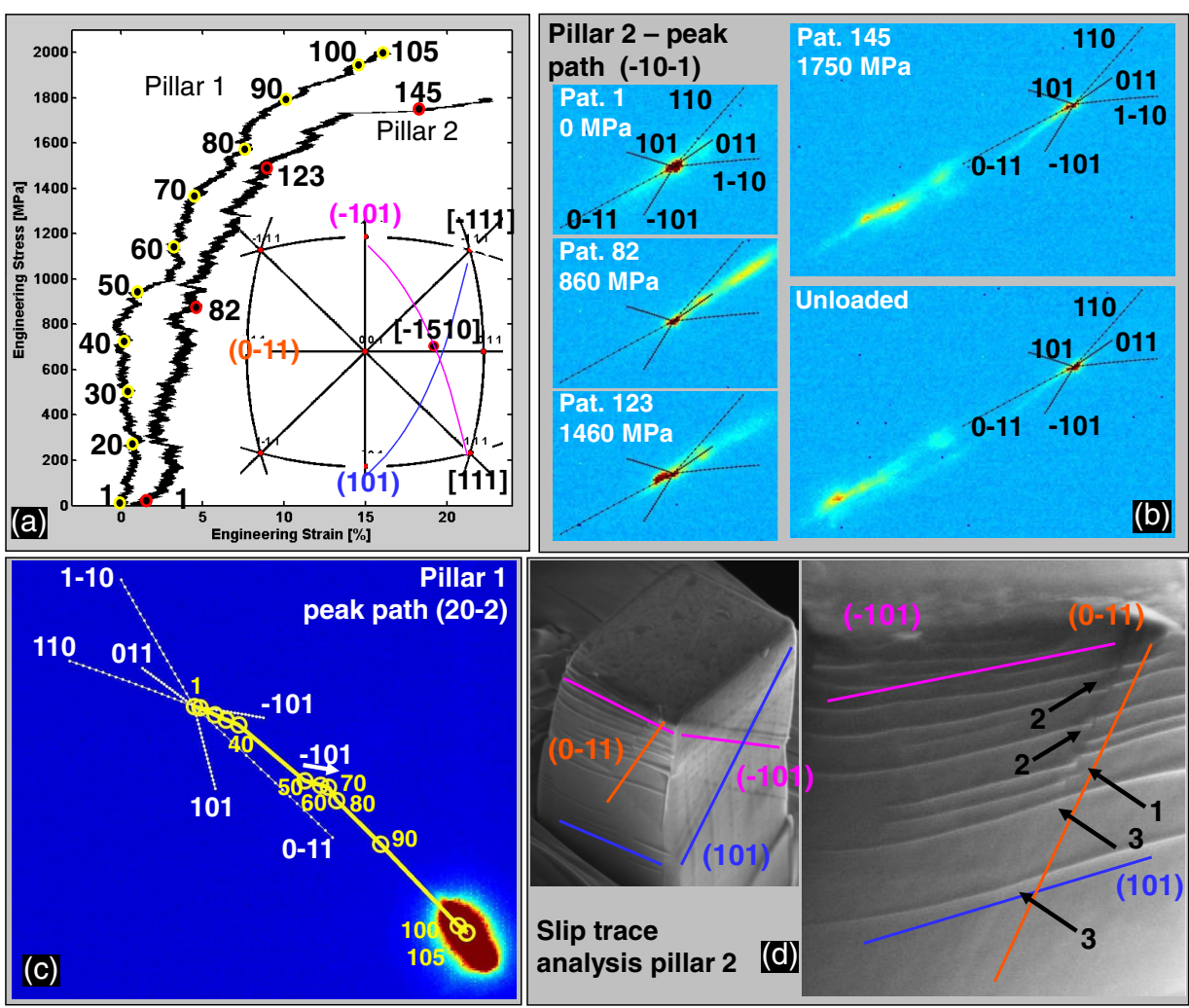

FIG. 1 (color online). In situ microcompression results. (a) Mechanical results of tungsten pillars 1 and 2. The inset represents the compression axis plotted in a stereographic projection with the slip systems of interest marked with a color code. The primary ( -101$)$, conjugate (101), and anomalous slip $(0-11)$ planes are indicated in purple, blue, and orange, respectively, the corresponding slip directions [111] and [-111] in bold black. (b) Two-dimensional representation of the $(-10-1)$ diffraction spot plotted together with the six possible $\{110\}$ rotation directions (black lines) of pillar 2, at five different stages of the load indicated on the stress-strain curve. (c) Path of the $(20-2)$ diffraction spot of pillar 1 with the six possible $\{110\}$ rotation lines plotted in white at different stages of the load indicated in (a): "1" for initial and "105" for final. (d) Slip traces on the surface of pillar 2 together with the indication of the corresponding planes. See text for more details.

change in the rotation direction occurs, which is ascribed to yielding. The peak moves back towards a direction indicative for slip on the anomalous $(0-11)$ plane till $23 \%$ strain. Such a rotation is very close to the (001), which may describe an equal active slip on the primary $(-101)$ and the conjugate (101) planes. Analysis of the other Laue reflections confirms these trends.

Figure 1(c) shows the peak path of the $(20-2)$ reflection spot during compression of pillar 1. Round circles represent its center of mass at stress levels marked on the stress-strain curve [Fig. 1(a)]. Until pattern 42 $(750 \mathrm{MPa})$, the peak moves slightly between the $(-101)$ and $(0-11)$ rotation lines. Then the peak path changes direction corresponding now to a rotation according to slip on the anomalous $(0-11)$, or to an equal amount of slip on (101) and (-101). The sudden change in movement observed between patterns 50 and 65 , where the rotation is due to single slip on $(-101)$, testifies that slip is not exclusively on the anomalous plane. Note that this region is also marked by a different hardening rate in the stress-strain curve.
Slip trace analysis is performed in the SEM and shown in Fig. 1(d) (pillar 2) and in Fig. S1 in the Supplemental Material [13] (pillar 1). In both cases, the surface exhibits multiple slip traces resulting from slip on the $(-101)$ primary plane (purple) and on the (101) conjugate plane (blue). Analyzing all sides of the pillar reveals more slip traces for the primary than the conjugate system. A zoom-in on the front face of pillar 2 shows a clear slip trace corresponding to the anomalous $(0-11)$ plane (orange). The step directions at the intersections between the anomalous, the primary, and the conjugate traces demonstrate activity on the primary slip system before (arrow 1), during (arrow 2), and after the anomalous slip trace was formed (arrow 3).

To summarize, Laue diffraction and slip trace analysis demonstrate that primary, conjugate, and anomalous slip systems are active. Coarse crystallographic slip traces occur first on the primary and the conjugate planes. At larger strains slip traces are also observed on the anomalous $(0-11)$ plane and strain production is alternated with the primary and secondary. These observations raise the 
fundamental question of how anomalous slip can be activated and lead to coarse crystallographic slip traces despite the fact that the shear stresses on the anomalous plane are too low to activate the motion of individual screw dislocations.

To analyze this question discrete dislocation dynamics (DDD) simulations [20-22] are performed on a tungsten micropillar in the same geometry as in the experiments. DDD simulations are based on elastic dislocation interactions and a constitutive law defining the stress-velocity relationship. Viscous damping for edge and mixed dislocations is assumed. Screw dislocation motion by kink pairs [23] is described by an Arrhenius law with a stress-dependent activation enthalpy determined from atomistic simulations $[6,24]$ which naturally incorporates non-Schmid effects. Purely based on the action of non-Schmid stresses, anomalous slip due to the motion of screw dislocations only cannot be explained in this orientation [25]. More details of the simulation setup are given in [13], where also the activation enthalpy for isolated screw dislocations on different glide systems are shown in Fig. S4 for the $\left[\begin{array}{lll}-1 & 5 & 10\end{array}\right]$ loading direction.

Figure 2 shows the total plastic slip activity in the compression direction. The lines indicate the orientations of the slip planes at the pillar surface using the color scheme of Fig. 1. The primary and conjugate slip systems contribute $42 \%$ and $31 \%$ to the total deformation. The anomalous slip plane $(0-11)$ contributes about $9 \%$ to the total deformation (Fig. S5 in the Supplemental Material [13]) with clear slip traces. The remaining plastic strain is contributed by the nine remaining systems and does not lead to visible slip traces in Fig. 2.

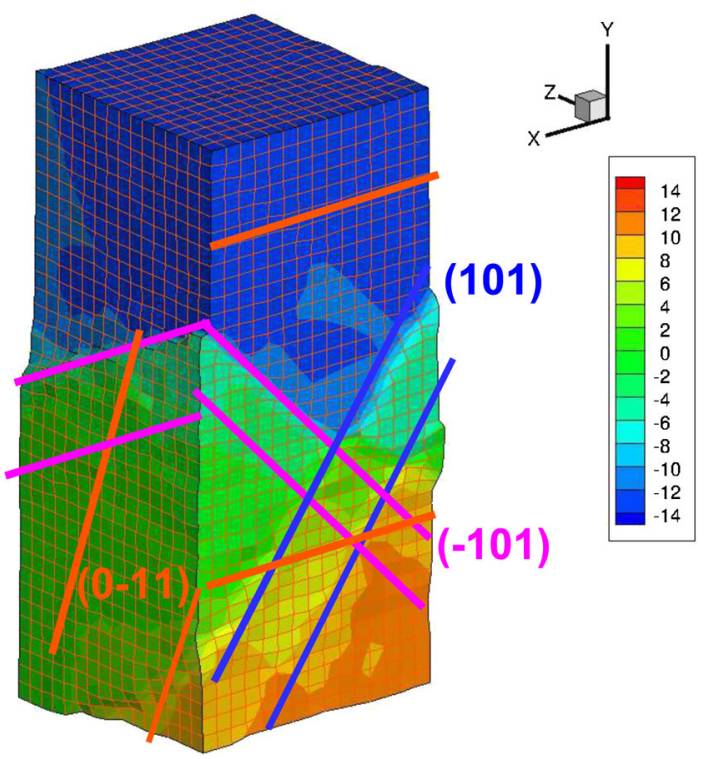

FIG. 2 (color online). The $y$ component of the total plastic displacement superimposed onto the deformed pillar after total plastic strain of $0.8 \%$ in units of the lattice constant. The deformations of the mesh are amplified by a factor of 15 .
Slip on both the primary and conjugate slip systems occurs primarily by motion of screw dislocations, as expected from the corresponding activation enthalpies. The slip directions [111] and [-111] are coplanar in the anomalous plane. Observed slip on this plane can therefore only result from the interaction of [111] and [-111] dislocations. Two mechanisms contribute to anomalous slip:

The first mechanism is stress-induced cross slip. Dislocation interactions can cause stress variations along both [111] and [-111] screw dislocations and impose a stress state, which favors cross slip via kink pair nucleation on the anomalous plane. This local activation does occur (see Fig. S6 [13]) but is short-lived and the screw part on the anomalous plane will cross slip back to a plane parallel to the initial plane, once the local stress is no longer favorable. This mechanism contributes to about $40 \%$ of the anomalous slip, but does not generate extended slip traces. It also helps to generate localized slip on the other nine slip systems.

A second mechanism is identified in our simulations only on the anomalous plane. Stress induced kinks on different $\{110\}$ planes can migrate towards each other along the screw dislocation and form a so-called cross kink. Figure 3 shows snapshots extracted from the 3D simulations. Two kink segments $A B$ and $B C$ make a sharp angle with each other and form the cross kink. $A B$ lies on the anomalous plane, $B C$ on the conjugate. The kinks have in general different lengths and experience therefore different driving forces, but they are geometrically constrained via the node $B$ to glide together. Both nodes $A$ and $B$ are kinematically constrained to move along the Burgers vector direction. Their effective direction of motion depends therefore on the resultant force: in the particular case in Fig. 3(a), the kink segment $A B$ glides on the anomalous plane against the direction in which it would want to move if it would have not been connected to segment $B C$.

When node $A$ reaches the surface [Fig. 3(b)] it is free to glide along the intersection line of the surface with the anomalous plane. Since the Peach-Koehler force on the segment $A B$ on the anomalous plane points inward, it increases its length when $A$ glides along the surface on the anomalous plane [Fig. 3(c)]. As schematically shown in Fig. 3(d), the motion of the dislocation segment $A B$ resembles a single armed dislocation source sweeping a large area on the anomalous plane before it can again attain screw orientation and possibly cross slip back to the lowactivation enthalpy plane. Since this mechanism produces slip by the motion of mixed dislocations on the anomalous plane, it generates coarse slip traces over a large region of the surface.

Previous models envision anomalous slip due to (a) image forces favoring perpetual kink nucleation on the anomalous plane [26] and (b) suppression of screw dislocation motion on primary and conjugate systems [27,28] while their 

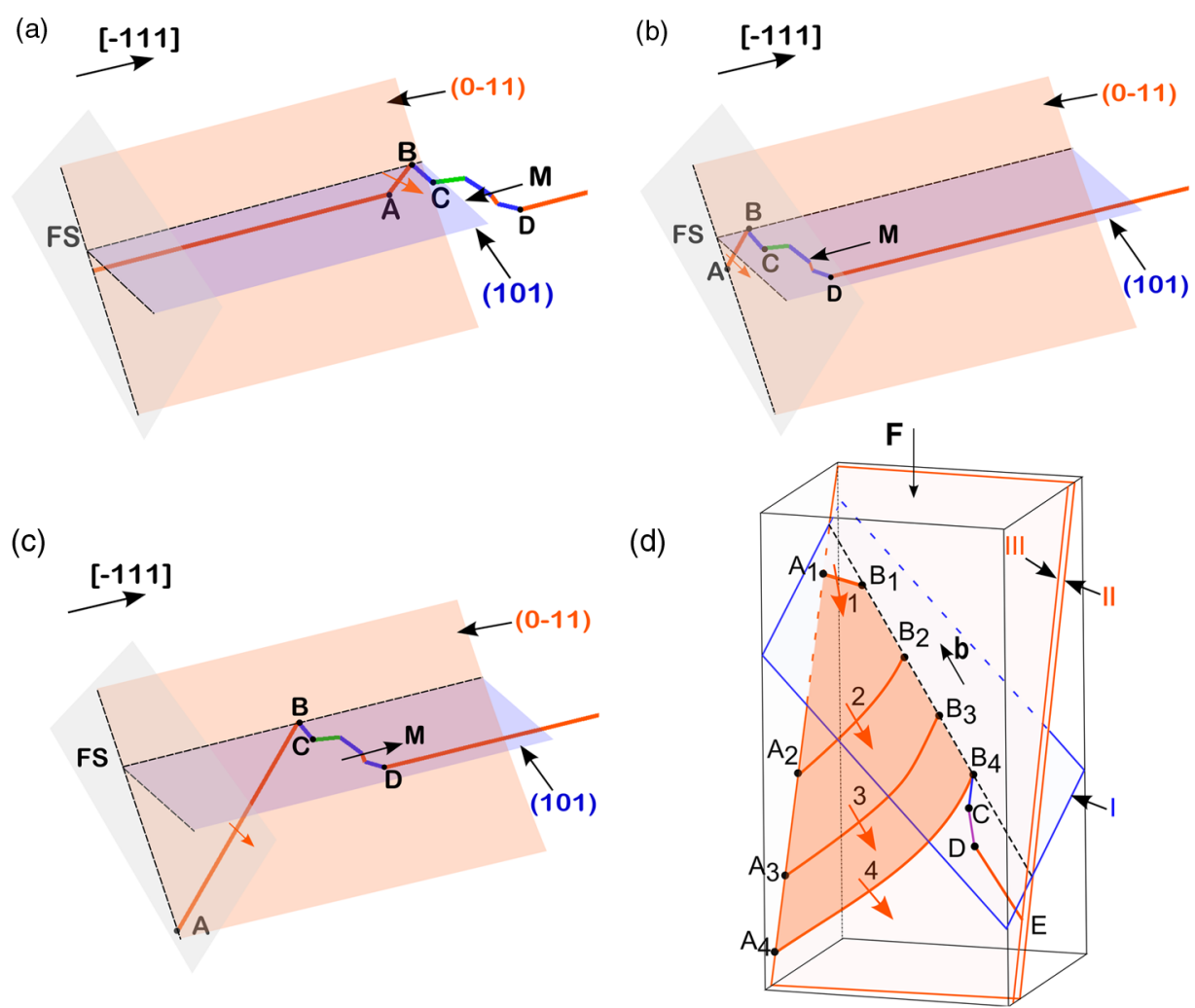

FIG. 3 (color online). Mechanism of anomalous slip. Snapshots [(a)-(d)] of small sections of our sample showing the generation of anomalous slip due to $[-111]$ dislocations on the $(0-11)$ plane. (a) scenario where the segments $A B$ and $B C$ form a cross kink. $A B$ lies on the $(0-11)$ plane, $B C$ on the (101) plane, and the remaining kink complex $M$ lies on different planes of the zone of the $[-111]$ Burgers vector. The free surface (FS) of the pillar is indicated in gray color. The glide planes are indicated in orange (anomalous) and blue (conjugate). Dislocation lines are colored according to their habit planes. Node $A$ is attached to a screw dislocation while node $B$ is connected to a section of several small mixed segments lying over several planes. The resultant direction of motion of the entire complex is indicated with the arrow $M$. In (b) node $A$ reaches the surface and is released from the screw dislocation. The dislocation section $A B$ can rapidly increase in length, generating slip on the anomalous plane shown in (c). (d) an overlay of the dislocation structure at four different time steps. The positions of the nodes $A$ and $B$ at the different time steps are marked as $A_{1}$ to $A_{4}$ and $B_{1}$ to $B_{4}$, respectively. The mixed segment $A B$ glides back in the direction of the orange arrow. Node $A$ moves along the intersection line of the anomalous plane II with the surface and $B$ moves along the screw direction given by the intersection of planes I and II. The segment $D E$ is screw oriented and lies on plane III (which is parallel to II). Segment $B C$ moves on plane I and $C D$ represents schematically the kink complex attached to $B C$ and moves noncrystallographically. The area swept by segment $A B$ on the anomalous plane II between time steps 1 to 4 is shown in orange.

continual interactions favor cooperative motion of screw dislocations on the anomalous plane. Neither can be identified in our simulations. Hsiung [29] advocates a dislocation interaction based activation of anomalous slip planes similar to our mechanism 1 , which by itself does not contribute significantly to the total slip.

None of the previous models can explain our observations of anomalous slip as a persistent mechanism in the presence of visible slip traces also on the primary and/or conjugate planes. On the contrary, our new mechanism for anomalous slip, which is based on cross-kink formation within the volume and the motion of mixed dislocations on the anomalous plane, actually requires slip and dislocationdislocation interaction on the primary and conjugate planes. The most important feature of the experimentally observed characteristics of anomalous slip, i.e., coarse crystallographic slip traces over a large specimen area, is a natural consequence of the cross-kink mechanism since it is caused by glide of mixed dislocations which cannot leave their glide planes. It inherently is a multidislocation process and cannot be explained from single dislocation behavior. This explains the ambiguous reporting in the past of concurrently primary and/or conjugate slip traces [9-12]. It explains as well why in our W samples anomalous slip traces are observed after and during primary (dominant) and conjugate slip.

This mechanistic understanding provides a completely new starting point for the formulation of crystal plasticity descriptions for low temperature deformation in bcc metals, which hitherto had not been able to include anomalous slip in any meaningful way. The cross-kink mechanism clearly leads to ways of linking slip activity on different slip 
systems and may therefore provide an essential ingredient to improved dislocation-based crystal plasticity formulations.

This new view on anomalous slip provides a new conceptual basis for the design of stronger bcc metals as well as an explanation for solute softening. Since mixed dislocations are involved in anomalous slip, the mechanism can, once initiated, operate at moderate stresses. A mechanistically guided approach in the search for efficient alloying elements to increase strength is therefore to make the motion of the cross kinks towards the surface more difficult. This can possibly be achieved by either putting obstacles in the way of the cross kinks, i.e., segregating pinning obstacles to the screw dislocations themselves, or by making kink formation easier so that plenty of cross kinks are generated but all of them will necessarily be short and therefore more or less immobile. For a rational approach to such alloying, one may take guidance from the experimental evidence that anomalous slip is suppressed by particular impurities and alloying elements [7,26,30]. Finally, the new anomalous mechanism involves the surface of the sample, and therefore should depend on system size, but controversial observations are reported [31,32].

C. M., H. V.S., and S. V.P. are grateful to S. Pierret, M. Weisser, C. Le Bourlot, A. Irastorza, S. Zabihzadeh, and Z. Sun for help during beam time at the Swiss Light Source. D. Grolimund and M. Williman from the MicroXAS/SLS are acknowledged for their high-quality technical support. H. V. S., C. M., and S. V. P. thank the Swiss National Science Foundation for the financial support. K. S., D. W., and P. G. acknowledge the Deutsche Forschungsgemeinschaft Grant No. Gu367/30. The authors thank C. Le Bourlot and R. Gröger for fruitful discussions.

C. M. and K. S. contributed equally to the research done in this paper. C. M. and S. V. P. performed the experiment, K. S. the simulations. C. M., S. V. P., and H. V. S. analyzed and interpreted the experiment. K. S., D. W., and P.G. analyzed and interpreted the simulation. D. G. acted as responsible beam scientist. C. M., K. S., D. W., S. V.P., P. G., and H. V. S. wrote the paper together.

[1] E. Schmid and W. Boas, Kristallplastizität. (Springer Verlag, Berlin, 1935).

[2] P. B. Hirsch, in Proceedings of the Fifth International Conference on Crystallography, Cambridge, 1960 (Cambridge Press, Cambridge, 1960).

[3] V. Vitek, R. J. Perrin, and D. K. Bowen, Philos. Mag. 21, 1049 (1970).

[4] M. S. Duesbery, Contemp. Phys. 27, 145 (1986).

[5] Z. M. Chen, M. Mrovec, and P. Gumbsch, Model. Simul. Mater. Sci. Eng. 21, 055023 (2013).
[6] R. Gröger, A. G. Bailey, and V. Vitek, Acta Mater. 56, 5401 (2008).

[7] J. W. Christian, Metall. Trans. A 14, 1237 (1983).

[8] M. S. Duesbery and R. A. Foxall, Philos. Mag. 20, 719 (1969).

[9] H. Matsui and H. Kimura, Mater. Sci. Eng. 24, 247 (1976).

[10] A. J. Garratt-reed and G. Taylor, Philos. Mag. A 39, 597 (1979).

[11] L. Kaun, A. Luft, J. Richter, and D. Schulze, Phys. Status Solidi 26, 485 (1968).

[12] W. Wasserbaech, Phys. Status Solidi A 147, 417 (1995).

[13] See Supplemental Material at http://link.aps.org/ supplemental/10.1103/PhysRevLett.113.025501 for details on pillar properties, the Laue diffraction experiment, kink-pair nucleation mechanism, and a discussion on the contribution of primary and conjugate slip systems and on stress-induced cross slip.

[14] H. Van Swygenhoven and S. Van Petegem, JOM 62, 36 (2010).

[15] D. M. Norfleet, D. M. Dimiduk, S. J. Polasik, M. D. Uchic, and M. J. Mills, Acta Mater. 56, 2988 (2008).

[16] W. F. Hosford, The Mechanics of Crystals and Textured Polycrystals (Oxford University Press, Oxford, 1993).

[17] R. I. Barabash, G. E. Ice, M. Kumar, and B. J. Ilavsky, Int. J. Plast. 25, 2081 (2009).

[18] B. C. Larson and L. E. Levine, J. Appl. Crystallogr. 46, 153 (2013).

[19] J. Zimmerman, H. Van Swygenhoven, C. Marichal, S. Van Petegem, C. Borca, B. Bartova, E. Oveisi, and C. Hebert, Acta Mater. 60, 4604(2012).

[20] D. Weygand, L. H. Friedman, E. van der Giessen, and A. Needleman, Model. Simul. Mater. Sci. Eng. 10, 437 (2002).

[21] D. Weygand and P. Gumbsch, Mater. Sci. Eng. A 400-401, 158 (2005).

[22] D. Weygand et al., High Performance Computing in Science and Engineering '08, edited by W. E. Nagel, D. B. Kröner, and M. M. Resch (Springer-Verlag, Berlin, 2008), pp. 507-523.

[23] B. Sestak and A. Seeger, Z. Metallkd. 69, 195 (1978).

[24] K. Srivastava, R. Gröger, D. Weygand, and P. Gumbsch, Int. J. Plast. 47, 126 (2013).

[25] R. Gröger, V. Racherla, J. L. Bassani, and V. Vitek, Acta Mater. 56, 5412 (2008).

[26] H. Matsui and H. Kimura, Scr. Metall. 7, 905 (1973).

[27] H. Matsui and H. Kimura, Scr. Metall. 9, 971 (1975).

[28] F. Louchet and L. P. Kubin, Acta Metall. 23, 17 (1975).

[29] L. Hsiung, Mater. Sci. Eng. A 528, 329 (2010).

[30] P. J. Jeffcoat, B. L. Mordike, and K. D. Rogausch, Philos. Mag. 34, 583 (1976).

[31] A. S. Schneider, D. Kaufmann, B. Clark, C. Frick, P. Gruber, R. Mönig, O. Kraft, and E. Arzt, Phys. Rev. Lett. 103, 105501 (2009).

[32] J. Kim, D. Jang, and J. R. Greer, Acta Mater. 58, 2355 (2010). 